\title{
CONICAL DIFFERENTIABILITY FOR BONE REMODELING CONTACT ROD MODELS *
}

\author{
Isabel N. Figueiredo ${ }^{1}$, Carlos F. Leal $^{1}$ and Cecília S. Pinto ${ }^{2}$
}

\begin{abstract}
We prove the conical differentiability of the solution to a bone remodeling contact rod model, for given data (applied loads and rigid obstacle), with respect to small perturbations of the cross section of the rod. The proof is based on the special structure of the model, composed of a variational inequality coupled with an ordinary differential equation with respect to time. This structure enables the verification of the two following fundamental results: the polyhedricity of a modified displacement constraint set defined by the obstacle and the differentiability of the two forms associated to the variational inequality.
\end{abstract}

Mathematics Subject Classification. 49J15, 49J20, 74B20, 74K10, 74L15, 90C31.

Received June 29, 2004.

\section{INTRODUCTION}

We consider a bone remodeling model, for a rod that may come into contact without friction with a rigid obstacle, due to the action of external loads, and we characterize the conical differentiability of the solution to this model, with respect to small variations of the geometry of the cross section of the rod. The knowledge of this conical differentiability is important, for example, in shape optimization bone remodeling problems, where the purpose is to control the geometry of the rod. In this introduction we describe the model and summarize the essential results of this paper.

Let $s \in[0, \delta], \delta>0$, be a small parameter and $\left.\Omega_{s}=\omega_{s} \times\right] 0, L[$ a domain, representing the reference configuration of a rod with cross section $\omega_{s} \subset \mathbb{R}^{2}$ and axis length $L>0$. For each $s \in[0, \delta], \omega_{s}=\omega+s \theta(\omega)$ is a perturbation of $\omega \subset \mathbb{R}^{2}$ in the direction of the vector field $\theta=\left(\theta_{1}, \theta_{2}\right): \mathbb{R}^{2} \longrightarrow \mathbb{R}^{2}$, that is regular enough. Consequently, the set $\Omega_{s}$ is a perturbation of the $\left.\operatorname{rod} \Omega=\Omega_{0}=\omega \times\right] 0, L[$. Let $V$ be a Hilbert space, representing the admissible displacements of the rod and $K \subset V$ a convex and closed subset of $V$, defining the constraints imposed on the admissible displacements of the rod. This set $K$ represents the possible contact, without friction, of the rod with the rigid obstacle. Let $<.,>$ denote the duality between $V^{\prime}$ and $V$, where $V^{\prime}$ is the dual of $V$, let $x=\left(x_{1}, x_{2}, x_{3}\right)$ be a generic element of $\bar{\Omega}$, and let $t$ be the time variable in the interval $[0, T]$, with $T>0$ a

\footnotetext{
Keywords and phrases. Adaptive elasticity, functional spaces, polyhedric set, rod.

* This work is part of the project "New materials, adaptive systems and their nonlinearities; modelling, control and numerical simulation" carried out in the framework of the european community program "Improving the human research potential and the socio-economic knowledge base" (HRN-CT-2002-00284).

1 Departamento de Matemática, Universidade de Coimbra, Apartado 3008, 3001-454 Coimbra, Portugal;

isabel.figueiredo@mat.uc.pt; carlosl@mat.uc.pt

2 Departamento de Matemática, Escola Superior de Tecnologia de Viseu, Campus Politécnico 3504-510 Viseu, Portugal; cagostinho@mat.estv.ipv.pt
} 
real positive parameter. Given a function $g^{s}(x, t)$, depending on $s \in[0, \delta]$ and defined in $\bar{\Omega} \times[0, T]$, we denote by $\dot{g}^{s}$ and $\partial_{i} g^{s}$ its partial derivatives, with respect to time $t$ and to $x_{i}$ for $i=1,2,3$, respectively.

For each perturbed $\operatorname{rod} \Omega_{s}$, with $s \in[0, \delta]$, the bone remodeling rod model that we consider is the elastic adaptive reduced rod model derived by Figueiredo and Trabucho [5], but with different boundary conditions and additional constraints (we recall that the theory of adaptive elasticity was introduced by Cowin and Hegedus $[2,7]$ and describes the physiological process of bone remodeling, that is, the continual process of growth, reinforcement, deposition and absorption of material, which occurs in living bone). Moreover, the bone remodeling model that we adopt in this paper, can be mathematically justified by the asymptotic expansion method as in Figueiredo and Trabucho [5] (cf. also Trabucho and Viãno [13], for an explanation of the asymptotic expansion method applied to elastic rod contact models), and is defined by the following system, formulated in the set $\bar{\Omega} \times[0, T]$ independent of $s(c f .(1.9))$

$$
\left[\begin{array}{l}
\text { Find } \quad\left(u^{s}, d^{s}\right) \quad \text { such that: } \\
u^{s}=\left(u_{1}^{s}, u_{2}^{s}, u_{3}^{s}\right): \bar{\Omega} \times[0, T] \rightarrow \mathbb{R}^{3} \quad \text { and } \quad d^{s}: \bar{\Omega} \times[0, T] \rightarrow \mathbb{R}, \\
u^{s}(., t) \in K \subset V, \\
a_{d^{s}}\left(u^{s}, v-u^{s}\right) \geq\left\langle L_{d^{s}}, v-u^{s}\right\rangle, \quad \forall v \in K \subset V, \\
\dot{d}^{s}=h\left(s, \theta, d^{s}, u^{s}\right), \quad \text { in } \Omega \times(0, T), \\
d^{s}(x, 0)=\bar{d}^{s}(x), \quad \text { in } \bar{\Omega} .
\end{array}\right.
$$

The pair $\left(u^{s}, d^{s}\right)$ is the unknown of the model: the vector field $u^{s}(., t)$ represents the displacement of the rod $\Omega$ at time $t$ and the scalar field $d^{s}(., t)$ is the measure of change in volume fraction of the elastic material of the $\operatorname{rod} \Omega$ at time $t$ (from a reference volume fraction of elastic material present in the porous bone, identified with the set $\Omega$ ). The variational inequality, where $a_{d^{s}}(.,$.$) is a bilinear form and L_{d^{s}}$ a linear form that depend on $d^{s}$, expresses the equilibrium of the rod $\Omega$ under the action of external forces, and subjected to the displacement constraints defined by the set $K$, that represents the possible contact of the rod with the rigid obstacle. The ordinary differential equation with respect to time, where $h$ is a function that depends on $u^{s}, d^{s}, \theta$ and $s$ (cf. (1.9) and (1.17)), is the so-called remodeling rate equation and models the physiological process of bone remodeling - if $\dot{d}^{s}$ is positive (respectively negative) it means that the volume fraction of elastic material is increasing (respectively decreasing). The unknowns $u^{s}$ and $d^{s}$ are interdependent: the displacement $u^{s}$ is the solution of the variational inequality and depends on $d^{s}$ and the unknown $d^{s}$ depends on $u^{s}$ and is the solution of the ordinary differential equation with respect to time.

The aim is to analyze the right-derivative of the solution to problem (0.1), with respect to $s$, at $s=0$. To compute this derivative we mainly use the regularity hypotheses for the solution to problem (0.1), convenient a priori norm bound estimates for the families $\left\{\left(u^{s}, d^{s}\right)\right\}_{s>0}$ and $\left\{\left(\frac{u^{s}-u^{0}}{s}, \frac{d^{s}-d^{0}}{s}\right)\right\}_{s>0}$, where $\left(u^{0}, d^{0}\right)$ is the solution to problem (0.1) with $s=0$, Theorem 4.14 of Sokolowski and Zolesio [12], p. 178 (or equivalentely, Th. 4.30 of Sokolowski and Zolesio [12] p. 210), the Schauder's fixed point theorem and uniqueness results. We remark that, in order to be able to apply the above mentioned Theorem 4.14 of Sokolowski and Zolesio [12], p. 178, we prove the polyhedricity of a modified constraint displacement set, using a technique described in Sokolowski and Zolesio [12], p. 209, and assuming an appropriate additional condition imposed to a non-negative Radon measure, as indicated in Proposition 3.4.

The main theorem of the paper can be formulated as follows.

Theorem 0.1. For each $t \in[0, T]$, let $A_{s}(., t) \in \mathcal{L}\left(V ; V^{\prime}\right)$ be the linear operator defined by $\left\langle A_{s} v, u\right\rangle=a_{d^{s}}(v, u)$ for all $v, u$ in $V$. Then the following three statements i), ii) and iii) are verified.

i) For each $t \in[0, T]$, there exists $A^{\prime}(., t) \in \mathcal{L}\left(V ; V^{\prime}\right)$ such that

$$
\lim _{s \rightarrow 0^{+}}\left\|\left(\frac{A_{s}-A_{0}}{s}-A^{\prime}\right)(., t)\right\|_{\mathcal{L}\left(V ; V^{\prime}\right)}=0 .
$$


ii) For each $t \in[0, T]$, there exists $L^{\prime}(., t) \in V^{\prime}$ such that

$$
\lim _{s \rightarrow 0^{+}}\left\|\left(\frac{L_{d^{s}}-L_{d^{0}}}{s}-L^{\prime}\right)(., t)\right\|_{V^{\prime}}=0 .
$$

iii) With the hypothesis of Proposition 3.4, for each time $t \in[0, T]$ the solution $\Pi\left(L_{d^{0}}\right)(., t)$ of the variational inequality

$$
\left[\begin{array}{l}
u^{0}(., t)=\Pi\left(L_{d^{0}}\right)(., t), \\
a_{d^{0}}\left(u^{0}, v-u^{0}\right) \geq\left\langle L_{d^{0}}, v-u^{0}\right\rangle, \quad \forall v \in K \subset V,
\end{array}\right.
$$

is conical differentiable at $L_{d^{0}}(., t)$, that is

$$
\forall l \in V^{\prime}, \quad \Pi\left(L_{d^{0}}+s l\right)(., t)=\Pi\left(L_{d^{0}}\right)(., t)+s Q(l)(., t)+o(s)
$$

for all $s>0$, small enough, where for each $t$, the mapping $Q(., t): V^{\prime} \rightarrow V$ is continuous and positively homogeneous and $\frac{\|o(s)\|_{V}}{s} \rightarrow 0$, as $s \rightarrow 0^{+}$.

Consequently, the properties i), ii) and iii) imply that, for each $t \in[0, T]$, the solution $\left(u^{s}, d^{s}\right)(., t)$ to the problem (0.1) is right-differentiable with respect to $s$, at $s=0$

$$
\begin{aligned}
& u^{s}(., t)=u^{0}(., t)+s u^{\prime}(., t)+o(s), \quad \text { in } \quad V, \quad \text { and } \quad u^{\prime}=Q\left(L^{\prime}-A^{\prime} u^{0}\right), \\
& d^{s}(., t)=d^{0}(., t)+s d^{\prime}(., t)+r(s), \quad \text { in } \quad L^{2}(\Omega),
\end{aligned}
$$

for all $s>0$, small enough, where $\left(u^{0}, d^{0}\right)$ is the solution of (0.1) for $s=0$ and as $s \rightarrow 0^{+}, \frac{\|o(s)\|_{V}}{s} \rightarrow 0$ and $\frac{\int_{\Omega} r(s) v \mathrm{~d} \Omega}{s} \rightarrow 0$, for any $v \in L^{2}(\Omega)$.

In particular $A^{\prime}$ and $L^{\prime}$ are defined by (2.25) and (2.26), $Q$ is defined by (3.28) and the pair $\left(u^{\prime}, d^{\prime}\right)$ is the unique solution of problem (5.1).

Finally let us briefly explain the contents of this paper. In Section 1 we introduce the family of bone remodeling rod models. In Sections 2 and 4 we give partial proofs of the conditions $(0.2)-(0.3)$ and $(0.6)-(0.7)$, respectively. In Section 3 we prove the property (0.5). Finally in Section 5 we completely prove Theorem 0.1.

\section{The FAMily OF ROD MODELS}

In this section we introduce some notations, definitions and hypotheses, we define the family of rod models depending on the parameter $s$, we redefine this family on a set independent of $s$ and finally we give some results concerning the existence and uniqueness of solution.

\subsection{Notations, definitions and hypotheses}

Let $\delta>0$ be a small parameter and for each $s \in[0, \delta]$ we consider the perturbation $I_{s}$ of the identity operator $I$ in $\mathbb{R}^{2}$, defined by $I_{s}\left(x_{1}, x_{2}\right)=(I+s \theta)\left(x_{1}, x_{2}\right)=\left(x_{s 1}, x_{s 2}\right)$, for all $\left(x_{1}, x_{2}\right) \in \mathbb{R}^{2}$, where $\theta=\left(\theta_{1}, \theta_{2}\right): \mathbb{R}^{2} \longrightarrow \mathbb{R}^{2}$ is a vector field regular enough (at least $\theta \in\left[W^{2, \infty}\left(\mathbb{R}^{2}\right)\right]^{2}$ ). Let $\omega$ be an open, bounded and connected subset of $\mathbb{R}^{2}$, with a boundary $\partial \omega$ regular enough. For each $s \in[0, \delta]$ we define $\omega_{s}=I_{s}(\omega)$, which is the perturbation of $\omega$ in the direction of the vector field $\theta$. We also denote by $\bar{\Omega}_{s}$ the set occupied by a cylindrical rod, in its reference configuration, with length $L>0$ and cross section $\omega_{s}$, that is $\bar{\Omega}_{s}=\bar{\omega}_{s} \times[0, L]=I_{s}(\bar{\omega}) \times[0, L] \subset \mathbb{R}^{3}$. Moreover we denote by $x_{s}=\left(x_{s 1}, x_{s 2}, x_{3}\right)$ a generic element of $\bar{\Omega}_{s}$ and define the sets $\left.\Gamma_{s}=\partial \omega_{s} \times\right] 0, L\left[, \Gamma_{s 0}=\bar{\omega}_{s} \times\{0\}\right.$, $\Gamma_{s L}=\bar{\omega}_{s} \times\{L\}$, where $\partial \omega_{s}$ is the boundary of $\omega_{s}$. These three sets represent, respectively, the lateral boundary of the $\operatorname{rod} \bar{\Omega}_{s}$ and its extremities. We assume that the boundary $\partial \omega_{s}$ is divided into two nonempty disjoint parts denoted by $\partial \omega_{s c}$ and $\partial \omega_{s g}$ and consequently we denote $\left.\Gamma_{s c}=\partial \omega_{s c} \times\right] 0, L\left[\right.$ and $\left.\Gamma_{s g}=\partial \omega_{s g} \times\right] 0, L[$. 
We assume that, for each $s \in[0, \delta]$ the coordinate system $\left(O, x_{s 1}, x_{s 2}, x_{3}\right)$ is a principal system of inertia associated with the rod $\Omega_{s}$. Consequently, axis $O x_{3}$ passes through the centroid of each section $\omega_{s} \times\left\{x_{3}\right\}$ and we have $\int_{\omega_{s}} x_{s 1} \mathrm{~d} \omega_{s}=\int_{\omega_{s}} x_{s 2} \mathrm{~d} \omega_{s}=\int_{\omega_{s}} x_{s 1} x_{s 2} \mathrm{~d} \omega_{s}=0$ (we observe that the choice of the vector field $\theta$, that realizes the shape variation of the cross section $\omega$, must be admissible with this last condition).

The set $C^{m}\left(\bar{\Omega}_{s}\right)$ stands for the space of real functions $m$ times continuously differentiable in $\bar{\Omega}_{s}$. The spaces $H^{m}\left(\Omega_{s}\right)=W^{m, 2}\left(\Omega_{s}\right)$ and $W^{0,2}\left(\Omega_{s}\right)=L^{2}\left(\Omega_{s}\right)$ are the usual Sobolev spaces, where $m \geq 0$ is a positive integer. The norms in these Sobolev spaces are denoted by $\|\cdot\|_{W^{m, 2}\left(\Omega_{s}\right)}$.

Throughout the paper, the latin indices $i, j, k, l \ldots$ belong to the set $\{1,2,3\}$, the greek indices $\alpha, \beta, \mu \ldots$ vary in the set $\{1,2\}$ and the summation convention with respect to repeated indices is employed, that is, for example, $a_{i} b_{i}=\sum_{i=1}^{3} a_{i} b_{i}$.

Let $T>0$ be a real parameter and we denote by $t$ the time variable in the interval $[0, T]$. If $V$ is a topological vectorial space, the set $C^{m}([0, T] ; V)$ is the space of functions $g: t \in[0, T] \rightarrow g(t) \in V$, such that $g$ is $m$ times continuously differentiable with respect to $t$. If $V$ is a Banach space we denote $\|\cdot\|_{C^{m}([0, T] ; V)}$ the usual norm in $C^{m}([0, T] ; V)$. Moreover, given a function $g_{s}\left(x_{s}, t\right)$ defined in $\bar{\Omega}_{s} \times[0, T]$ we denote by $\dot{g}_{s}$ its partial derivative with respect to time, by $\partial_{s \alpha} g_{s}$ and $\partial_{3} g_{s}$ its partial derivatives with respect to $x_{s \alpha}$ and $x_{3}$, that is, $\dot{g}_{s}=\frac{\partial g_{s}}{\partial t}$, $\partial_{s \alpha} g_{s}=\frac{\partial g_{s}}{\partial x_{s \alpha}}$ and $\partial_{3} g_{s}=\frac{\partial g_{s}}{\partial x_{3}}$.

For each $s \in[0, \delta]$ we consider the following model for the rod $\Omega_{s}$, that can be mathematically justified by the asymptotic expansion method as in Figueiredo and Trabucho [5].

$$
\left[\begin{array}{l}
\text { Find } \quad\left(u_{s}, d_{s}\right) \quad \text { such that: } \\
u_{s}=\left(u_{s 1}, u_{s 2}, u_{s 3}\right): \bar{\Omega}_{s} \times[0, T] \rightarrow \mathbb{R}^{3} \quad \text { and } \quad d_{s}: \bar{\Omega}_{s} \times[0, T] \rightarrow \mathbb{R}, \\
u_{s}(., t) \in K_{s} \subset V_{s}, \\
a_{d_{s}}\left(u_{s}, v_{s}-u_{s}\right) \geq\left\langle L_{d_{s}}, v_{s}-u_{s}\right\rangle, \quad \forall v_{s} \in K_{s} \subset V_{s}, \\
\dot{d}_{s}=b\left(d_{s}\right)+c\left(d_{s}\right) e_{33}\left(u_{s}\right), \quad \text { in } \quad \Omega_{s} \times(0, T), \\
d_{s}(x, 0)=\bar{d}_{s}(x), \quad \text { in } \bar{\Omega}_{s} .
\end{array}\right.
$$

The unknowns of the model (1.1) are the displacement vector field $u_{s}\left(x_{s}, t\right)$, corresponding to the displacement of the point $x_{s}$ of the rod $\bar{\Omega}_{s}$ at time $t$ and the measure of change in volume fraction of the elastic material (from a reference volume fraction denoted in the sequel by $\left.\xi_{s 0}\right) d_{s}\left(x_{s}, t\right)$ at $\left(x_{s}, t\right)$. In particular $e_{33}\left(u_{s}\right)$ is an element of the linear strain tensor $\left(e_{i j}\left(u_{s}\right)\right)=\left(\frac{1}{2}\left(\partial_{s i} u_{s j}+\partial_{s j} u_{s i}\right)\right)$, and it is a function of $u_{s}$.

On the other hand, the data of the model (1.1) are the following: the space $V_{s}$ of admissible displacements, the set $K_{s} \subset V_{s}$ of displacement constraints, the bilinear form $a_{d_{s}}(.,):. V_{s} \times V_{s} \rightarrow \mathbb{R}$ and the element $L_{d_{s}}(.) \in$ $V^{\prime}$, that depend on the unknown $d_{s}$ and represent, respectively, the elastic equilibrium equations and the external forces acting on the rod, the initial value of the change in volume fraction $\bar{d}_{s}()=.d_{s}(., 0)$, and the coefficients $b\left(d_{s}\right)$ and $c\left(d_{s}\right)$ which are material coefficients depending upon the change in volume fraction $d_{s}$.

Assuming that the rod is clamped at its extremities $\Gamma_{s 0}=\bar{\omega}_{s} \times\{0\}$ and $\Gamma_{s L}=\bar{\omega}_{s} \times\{L\}$, the space $V_{s}$ of admissible displacements is defined by

$$
V_{s}=\left\{v_{s} \in\left[W_{0}^{2,2}(] 0, L[)\right]^{2} \times W^{1,2}\left(\Omega_{s}\right): \quad e_{\alpha \beta}\left(v_{s}\right)=e_{3 \beta}\left(v_{s}\right)=0\right\}
$$

which is identified with the set

$$
\begin{aligned}
& \left\{v_{s}=\left(v_{s 1}, v_{s 2}, v_{s 3}\right) \in\left[W_{0}^{2,2}(] 0, L[)\right]^{2} \times W^{1,2}\left(\Omega_{s}\right): \quad v_{s \alpha}\left(x_{s}\right)=v_{s \alpha}\left(x_{3}\right),\right. \\
& \left.v_{s 3}\left(x_{s}\right)=\underline{v}_{s 3}\left(x_{3}\right)-x_{s \alpha} \partial_{3} v_{\alpha}\left(x_{3}\right), \quad \underline{v}_{s 3} \in W_{0}^{1,2}(] 0, L[)\right\},
\end{aligned}
$$


that is, $V_{s} \subset\left[W^{1,2}\left(\Omega_{s}\right)\right]^{3}$ is the space of Bernoulli-Navier displacements. We remark that $W_{0}^{1,2}(] 0, L[)=\{\xi \in$ $\left.W^{1,2}(] 0, L[): \xi(0)=\xi(L)=0\right\}$, and $W_{0}^{2,2}(] 0, L[)=\left\{\xi \in W^{2,2}(] 0, L[): \xi(0)=\xi(L)=0, \quad \xi^{\prime}(0)=\xi^{\prime}(L)=0\right\}$, where $\xi^{\prime}$ is the first derivative of $\xi$.

The bilinear form $a_{d_{s}}(.,$.$) is defined$

$$
a_{d_{s}}\left(u_{s}, v_{s}\right)=\int_{\Omega_{s}} \frac{1}{b_{3333}\left(d_{s}\right)} e_{33}\left(u_{s}\right) e_{33}\left(v_{s}\right) \mathrm{d} \Omega_{s}, \quad \forall u_{s}, v_{s} \in V_{s}
$$

where $e_{33}\left(v_{s}\right)=\partial_{3} v_{s 3}=\partial_{3} \underline{v}_{s 3}-x_{s \alpha} \partial_{33} v_{s \alpha}$ and $b_{3333}\left(d_{s}\right)$ is a material coefficient that depends on $d_{s}$ (in fact it is an element of the matrix $\left(b_{i j k l}\left(d_{s}\right)\right)$ which is the inverse of the matrix composed of the three-dimensional elastic coefficients of the rod $\bar{\Omega}_{s}$, as explained in Figueiredo and Trabucho [5]).

The element $L_{d_{s}}$ is defined by

$$
\left\langle L_{d_{s}}, v_{s}\right\rangle=\int_{\Omega_{s}} \gamma\left(\xi_{s 0}+P_{\eta}\left(d_{s}\right)\right) f_{s i} v_{s i} \mathrm{~d} \Omega_{s}+\int_{\Gamma_{s} g} g_{s i} v_{s i} \mathrm{~d} \Gamma_{s}, \quad \forall v_{s} \in V_{s},
$$

where $\gamma$ is the density of the full elastic material, which is supposed to be a constant independent of $s, \xi_{s 0}$ is the reference volume fraction of the elastic material (already mentioned immediately after the definition of the problem (1.1)) that belongs to $C^{1}\left(\bar{\Omega}_{s}\right), f_{s}=\left(f_{s i}\right)$ and $g=\left(g_{s i}\right)$ are, respectively, the density of body loads and normal tractions on the lateral boundary $\Gamma_{s g}$ of the $\operatorname{rod} \bar{\Omega}_{s}$, and $P_{\eta}($.$) is a truncation operator. We suppose$ that $0<\xi_{s 0}^{\min } \leq \xi_{s 0}\left(x_{s}\right) \leq \xi_{s 0}^{\max }<1$, for all $x_{s} \in \bar{\Omega}_{s}$, and the truncation operator $P_{\eta}$ is of class $C^{1}$ and satisfies $0<\frac{\eta}{2} \leq\left(\xi_{s 0}+P_{\eta}\left(d_{s}\right)\right)\left(x_{s}\right) \leq 1$ for all $x_{s} \in \bar{\Omega}_{s}$, where $\eta>0$ is a small parameter. We also assume that $f_{s i} \in C^{1}([0, T])$ and $g_{s i} \in C^{1}\left([0, T] ; W^{1-1 / p, p}\left(\Gamma_{s g}\right)\right)$, with $p>3$. These hypotheses of regularity on the forces are necessary to obtain existence results.

The set $K_{s} \subset V_{s}$ is a nonempty, closed and convex subset of $V_{s}$, representing the additional constraints imposed on the admissible displacements. Due to the action of the applied loads we assume that the lateral surface $\Gamma_{s c}$ of the rod may come into contact, without friction, with a rigid obstacle. Moreover, we suppose that the candidate contact surface $\Gamma_{s c}$ is plane and perpendicular to the inertia axis $O x_{s 1}$ of the rod. Therefore, from these assumptions we deduce that the set $K_{s}$ of the reduced elastic adaptive rod model (1.1) is of the form (cf. also Trabucho and Viãno [13], Chap. VI, p. $770(28.46)$ )

$$
K_{s}=\left\{v_{s} \in V_{s}: \quad v_{s 1} \geq \psi \quad \text { in }\right] 0, L[\}
$$

where $\psi:[0, L] \rightarrow \mathbb{R}$ is a smooth enough scalar function, such that $\psi\left(x_{3}\right)<0$, for all $x_{3} \in[0, L]$. Then the set $K_{s}$ physically imposes that the bending component $v_{s 1}$, of the admissible displacement $v_{s}$, can touch but not penetrate the obstacle represented by the function $\psi$.

Finally, we suppose that the initial value $\bar{d}_{s}()=.d_{s}(., 0)$ of the change in volume fraction verifies $\bar{d}_{s} \in C^{0}\left(\bar{\Omega}_{s}\right)$ and the material coefficients $b\left(d_{s}\right), c\left(d_{s}\right)$ and $b_{3333}\left(d_{s}\right)$ appearing in the right hand side of the remodeling rate equation are continuously differentiable with respect to $d_{s}$. In addition we also assume that there exist strictly positive constants $C_{1}, C_{2}, C_{3}, C_{4}, C_{5}$ and $C_{6}$ independent of $s$ and $t$ such that for any $\left(x_{s}, t\right) \in \Omega_{s} \times[0, T]$

$$
\begin{aligned}
& 0 \leq C_{1} \leq \frac{1}{b_{3333}\left(d_{s}\right)} \leq C_{2}, \quad \forall s \in[0, \delta], \\
& \left|b\left(d_{s}\right)\right| \leq C_{3}, \quad\left|b^{\prime}\left(d_{s}\right)\right| \leq C_{4}, \quad\left|c\left(d_{s}\right)\right| \leq C_{5}, \quad\left|c^{\prime}\left(d_{s}\right)\right| \leq C_{6}, \quad \forall s \in[0, \delta],
\end{aligned}
$$

where $b^{\prime}($.$) and c^{\prime}($.$) are the derivatives of the scalar functions b($.$) and c($.$) , respectively.$

We observe that we could have considered in (1.1) a remodeling rate equation depending nonlinearly on $e_{33}\left(u_{s}\right)$, that is (cf. Figueiredo and Trabucho [5])

$$
\dot{d}_{s}=b\left(d_{s}\right)+c\left(d_{s}\right) e_{33}\left(u_{s}\right)+\frac{1}{b_{3333}\left(d_{s}\right)} e_{33}\left(u_{s}\right) e_{33}\left(u_{s}\right)
$$


which is an equation that seems to be more suitable to represent the remodeling rate process, from the mechanical view-point, even in the case of small strains ( $c f$. Hegedus and Cowin [7]). In fact, all the results of Theorem 0.1 can also be derived for this type of nonlinear remodeling rate equation; the nonlinear term $\frac{1}{b_{3333}\left(d_{s}\right)} e_{33}\left(u_{s}\right) e_{33}\left(u_{s}\right)$ in (1.8) only originates more complicated calculus.

\subsection{The family of rod models formulated in $\Omega$}

In order to derive the results stated in Theorem 0.1 we reformulate now, for each $s \in[0, \delta]$, the problem (1.1) in the fixed $\operatorname{rod} \bar{\Omega}$ independent of $s$.

We consider the perturbation map $I_{s}$ defined in Section 1.1 that maps $\Omega$ onto $\Omega_{s}$. For a function $v_{s}$ defined in $\Omega_{s}$ we associate the corresponding function $v^{s}$ (with upper index $s$ ) defined in $\Omega$ by $v^{s}=v_{s} o I_{s}$. Performing this change of variables and observing that $e_{33}\left(v_{s}\right)=e_{33}\left(v^{s}\right)-s \theta_{\alpha} \partial_{33} v_{\alpha}^{s}$, for any $v_{s} \in V_{s}$, the problem (1.1) is equivalent to the following problem defined in the $\operatorname{rod} \Omega$ independent of $s$

$$
\left[\begin{array}{l}
\text { Find } \quad\left(u^{s}, d^{s}\right) \quad \text { such that: } \\
u^{s}=\left(u_{1}^{s}, u_{2}^{s}, u_{3}^{s}\right): \bar{\Omega} \times[0, T] \rightarrow \mathbb{R}^{3} \quad \text { and } \quad d^{s}: \bar{\Omega} \times[0, T] \rightarrow \mathbb{R}, \\
u^{s}(., t) \in K \subset V, \\
a_{d^{s}}\left(u^{s}, v-u^{s}\right) \geq\left\langle L_{d^{s}}, v-u^{s}\right\rangle, \quad \forall v \in K, \\
\dot{d}^{s}=b\left(d^{s}\right)+c\left(d^{s}\right) e_{33}\left(u^{s}\right)-s c\left(d^{s}\right) \theta_{\alpha} \partial_{33} u_{\alpha}^{s}, \quad \text { in } \quad \Omega \times(0, T), \\
d^{s}(x, 0)=\bar{d}(x), \quad \text { in } \bar{\Omega},
\end{array}\right.
$$

where we suppose that $\bar{d}$ is independent of $s \in[0, \delta]$, and, for all $u$ and $v$ in $V$

$$
a_{d^{s}}(u, v)=a_{0}^{s}(u, v)+s a_{1}^{s}(u, v)+s^{2} a_{2}^{s}(u, v)+s^{3} a_{3}^{s}(u, v)+s^{4} a_{4}^{s}(u, v),
$$

and

$$
\left\{\begin{aligned}
\left\langle L_{d^{s}}, v\right\rangle= & F_{0}^{s}(v)+G_{0}^{s}(v)+s\left(F_{1}^{s}(v)+G_{1}^{s}(v)\right) \\
& +s^{2}\left(F_{2}^{s}(v)+G_{2}^{s}(v)\right)+s^{3}\left(F_{3}^{s}(v)+G_{3}^{s}(v)\right) .
\end{aligned}\right.
$$

The bilinear forms $a_{i}^{S}(.,$.$) , for i=0,1,2,3,4$, are defined by

$$
\begin{aligned}
& a_{0}^{s}(u, v)=\int_{\Omega} \frac{1}{b_{3333}\left(d^{s}\right)} e_{33}(u) e_{33}(v) \mathrm{d} \Omega \\
& a_{1}^{s}(u, v)=\int_{\Omega} \frac{1}{b_{3333}\left(d^{s}\right)}\left[-\theta_{\alpha}\left(e_{33}(u) \partial_{33} v_{\alpha}+e_{33}(v) \partial_{33} u_{\alpha}\right)+e_{33}(u) e_{33}(v) \operatorname{div} \theta\right] \mathrm{d} \Omega, \\
& a_{2}^{s}(u, v)=\int_{\Omega} \frac{1}{b_{3333}\left(d^{s}\right)}\left[e_{33}(u) e_{33}(v) \operatorname{det} \nabla \theta+\theta_{\alpha} \theta_{\beta} \partial_{33} u_{\alpha} \partial_{33} v_{\beta}-(\operatorname{div} \theta) \theta_{\alpha}\left(e_{33}(u) \partial_{33} v_{\alpha}+e_{33}(v) \partial_{33} u_{\alpha}\right)\right] \mathrm{d} \Omega, \\
& a_{3}^{s}(u, v)=\int_{\Omega} \frac{1}{b_{3333}\left(d^{s}\right)}\left[\theta_{\alpha} \theta_{\beta} \partial_{33} u_{\alpha} \partial_{33} v_{\beta} \operatorname{div} \theta-(\operatorname{det} \nabla \theta) \theta_{\alpha}\left(e_{33}(u) \partial_{33} v_{\alpha}+e_{33}(v) \partial_{33} u_{\alpha}\right)\right] \mathrm{d} \Omega, \\
& a_{4}^{s}(u, v)=\int_{\Omega} \frac{1}{b_{3333}\left(d^{s}\right)}\left[\theta_{\alpha} \theta_{\beta} \partial_{33} u_{\alpha} \partial_{33} v_{\beta} \operatorname{det} \nabla \theta\right] \mathrm{d} \Omega .
\end{aligned}
$$


The forms $F_{j}^{s}(v)$ and $G_{j}^{s}(v)$, for $j=0,1,2,3$, are defined by

$$
\begin{aligned}
& F_{0}^{s}(v)=\int_{\Omega} \gamma\left(\xi_{0}^{s}+P_{\eta}\left(d^{s}\right)\right)\left(f_{\alpha}^{s} v_{\alpha}+f_{3}^{s} \underline{v}_{3}\right) \mathrm{d} \Omega, \\
& F_{1}^{s}(v)=\int_{\Omega} \gamma\left(\xi_{0}^{s}+P_{\eta}\left(d^{s}\right)\right)\left[\left(f_{\alpha}^{s} v_{\alpha}+f_{3}^{s} \underline{v}_{3}\right) \operatorname{div} \theta-f_{3}^{s} \theta_{\alpha} \partial_{3} v_{\alpha}\right] \mathrm{d} \Omega, \\
& F_{2}^{s}(v)=\int_{\Omega} \gamma\left(\xi_{0}^{s}+P_{\eta}\left(d^{s}\right)\right)\left[\left(f_{\alpha}^{s} v_{\alpha}+f_{3}^{s} \underline{v}_{3}\right) \operatorname{det} \nabla \theta-f_{3}^{s} \theta_{\alpha} \partial_{3} v_{\alpha} \operatorname{div} \theta\right] \mathrm{d} \Omega, \\
& F_{3}^{s}(v)=-\int_{\Omega} \gamma\left(\xi_{0}^{s}+P_{\eta}\left(d^{s}\right)\right) f_{3}^{s} \theta_{\alpha} \partial_{3} v_{\alpha} \operatorname{det} \nabla \theta \mathrm{d} \Omega,
\end{aligned}
$$

and

$$
\begin{aligned}
G_{0}^{s}(v) & =\int_{\Gamma_{g}}\left(g_{\alpha}^{s} v_{\alpha}+g_{3}^{s} \underline{v}_{3}\right) \mathrm{d} \Gamma \\
G_{1}^{s}(v) & =\int_{\Gamma_{g}}\left[\left(g_{\alpha}^{s} v_{\alpha}+g_{3}^{s} \underline{v}_{3}\right) G_{1}(\theta, n)-g_{3}^{s} \theta_{\alpha} \partial_{3} v_{\alpha}\right] \mathrm{d} \Gamma \\
G_{2}^{s}(v) & =\int_{\Gamma_{g}}\left[\left(g_{\alpha}^{s} v_{\alpha}+g_{3}^{s} \underline{v}_{3}\right) G_{2}(\theta, n)-g_{3}^{s} \theta_{\alpha} \partial_{3} v_{\alpha} G_{1}(\theta, n)\right] \mathrm{d} \Gamma \\
G_{3}^{s}(v) & =-\int_{\Gamma_{g}} g_{3}^{s} \theta_{\alpha} \partial_{3} v_{\alpha} G_{3}(\theta, n) \mathrm{d} \Gamma
\end{aligned}
$$

where $\Gamma=\Gamma_{0}, \Gamma_{g}=\Gamma_{0 g}$, and $G_{1}(\theta, n), G_{2}(\theta, n), G_{3}(\theta, n)$ are bounded scalar functions of $\theta$ and $n$ (the unit outer normal vector to the lateral boundary $\Gamma_{s}$ for $\left.s=0\right)$. The space $V$ is a subspace of $\left[H_{0}^{2}(] 0, L[)\right]^{2} \times H^{1}(\Omega)=$ $\left[W_{0}^{2,2}(] 0, L[)\right]^{2} \times W^{1,2}(\Omega)$ defined by

$$
\begin{aligned}
V=\left\{u \in\left[H_{0}^{2}(] 0, L[)\right]^{2} \times H^{1}(\Omega): \quad v=\right. & \left(v_{1}\left(x_{3}\right), v_{2}\left(x_{3}\right), v_{3}\left(x_{1}, x_{2}, x_{3}\right)\right), \\
& \left.v_{3}\left(x_{1}, x_{2}, x_{3}\right)=\underline{v}_{3}\left(x_{3}\right)-x_{\alpha} \partial_{3} v_{\alpha}\left(x_{3}\right), \text { with } \underline{v}_{3} \in H_{0}^{1}(] 0, L[)\right\} .
\end{aligned}
$$

We consider that $V$ is equipped with the usual norm of $\left[H^{1}(\Omega)\right]^{3}$. Finally, the closed convex $K$ is defined by

$$
K=\left\{v \in V: \quad v_{1}\left(x_{3}\right) \geq \psi\left(x_{3}\right), \quad \text { in } \quad\right] 0, L[\} .
$$

We remark that if we have considered the remodeling rate equation (1.8), then in (1.9) the ordinary differential equation would be the following

$$
\left[\begin{array}{rl}
\dot{d}^{s}= & c\left(d^{s}\right) e_{33}\left(u^{s}\right)+b\left(d^{s}\right)+\frac{1}{b_{3333}\left(d^{s}\right)} e_{33}\left(u^{s}\right) e_{33}\left(u^{s}\right) \\
& +s\left(\frac{-2}{b_{3333}\left(d^{s}\right)} \theta_{\alpha} \partial_{33} u_{\alpha}^{s} e_{33}\left(u^{s}\right)-c\left(d^{s}\right) \theta_{\alpha} \partial_{33} u_{\alpha}^{s}\right) \\
& +s^{2} \frac{1}{b_{3333}\left(d^{s}\right)}\left(\theta_{\alpha} \partial_{33} u_{\alpha}^{s}\right)\left(\theta_{\beta} \partial_{33} u_{\beta}^{s}\right), \quad \text { in } \quad \Omega \times(0, T) .
\end{array}\right.
$$


In the sequel we represent by $\left(u^{0}, d^{0}\right)$ the solution of problem (1.9) for $s=0$, that is:

$$
\left[\begin{array}{l}
\text { Find }\left(u^{0}, d^{0}\right) \quad \text { such that: } \\
u^{0}=\left(u_{1}^{0}, u_{2}^{0}, u_{3}^{0}\right): \bar{\Omega} \times[0, T] \rightarrow \mathbb{R}^{3} \quad \text { and } \quad d^{0}: \bar{\Omega} \times[0, T] \rightarrow \mathbb{R}, \\
u^{0}(., t) \in K \subset V, \\
a_{d^{0}}\left(u^{0}, v-u^{0}\right) \geq\left\langle L_{d^{0}}, v-u^{0}\right\rangle, \quad \forall v \in K, \\
\dot{d}^{0}=b\left(d^{0}\right)+c\left(d^{0}\right) e_{33}\left(u^{0}\right), \quad \text { in } \quad \Omega \times(0, T), \\
d^{0}(x, 0)=\bar{d}(x), \quad \text { in } \bar{\Omega},
\end{array}\right.
$$

where $a_{d^{0}}(.,$.$) and L_{d^{0}}($.$) are independent of s$ and defined by

$$
\begin{aligned}
a_{d^{0}}(z, v) & =\int_{\Omega} \frac{1}{b_{3333}\left(d^{0}\right)} e_{33}(z) e_{33}(v) \mathrm{d} \Omega \\
L_{d^{0}}(v) & =F_{0}(v)+G_{0}(v)
\end{aligned}
$$

where

$$
\begin{aligned}
F_{0}(v) & =\int_{\Omega} \gamma\left(\xi_{0}+P_{\eta}\left(d^{0}\right)\right)\left(f_{\alpha} v_{\alpha}+f_{3} \underline{v}_{3}\right) \mathrm{d} \Omega, \\
G_{0}(v) & =\int_{\Gamma_{g}}\left(g_{\alpha} v_{\alpha}+g_{3} \underline{v}_{3}\right) \mathrm{d} \Gamma,
\end{aligned}
$$

for all $z$ and $v$ in $V$, with $f=\left(f_{i}\right)$ and $g=\left(g_{i}\right)$ independent of $s$. For the case where the remodeling rate equation is defined by (1.17) then for $s=0$

$$
\dot{d}^{0}=b\left(d^{0}\right)+c\left(d^{0}\right) e_{33}\left(u^{0}\right)+\frac{1}{b_{3333}\left(d^{0}\right)} e_{33}\left(u^{0}\right) e_{33}\left(u^{0}\right), \quad \text { in } \quad \Omega \times(0, T) .
$$

We also observe that because of the following Korn's type inequality in the space $V$ (cf. Ciarlet [1] or Valent [14])

$$
\exists c>0: \quad\|v\|_{\left[H^{1}(\Omega)\right]^{3}}^{2} \leq c\left\|e_{33}(v)\right\|_{L^{2}(\Omega)}^{2}, \quad \forall v \in V
$$

where

$$
\left\|e_{33}(v)\right\|_{L^{2}(\Omega)}^{2}=\left\|\partial_{3} \underline{v}_{3}\right\|_{L^{2}(0, L)}^{2}+\left(\int_{\omega} x_{\alpha}^{2} \mathrm{~d} \omega\right)\left\|\partial_{33} v_{\alpha}\right\|_{L^{2}(0, L)}^{2} .
$$

Then $\left\|e_{33}(.)\right\|_{L^{2}(\Omega)}$ is a norm in the space $V$, equivalent to the usual norm induced in $V$ by $\|\cdot\|_{\left[H^{1}(\Omega)\right]^{3}}$. So in the sequel and for all $v \in V$, we denote by $\|v\|_{V}$ the norm $\left\|e_{33}(v)\right\|_{L^{2}(\Omega)}$ or the norm $\|v\|_{\left[H^{1}(\Omega)\right]^{3}}$. Moreover, $V$ is a Hilbert space with the norm $\left\|e_{33}(.)\right\|_{L^{2}(\Omega)}$ and for each $s$, the bilinear form $a_{0}^{s}(.,$.$) is continuous and elliptic in$ $V$ (this statement is also a consequence of the condition imposed on the coefficient $b_{3333}\left(d^{s}\right)$ in (1.7)), that is, there exist positive constants $C_{1}$ and $C_{2}$ independent of $s$, for all $z$ and $v$ in $V$ and for all $s \in[0, \delta]$, such that

$$
\begin{aligned}
& a_{0}^{s}(z, v) \leq C_{2}\left\|e_{33}(z)\right\|_{L^{2}(\Omega)}\left\|e_{33}(v)\right\|_{L^{2}(\Omega)}=C_{2}\|z\|_{V}\|v\|_{V} \text { (continuity), } \\
& a_{0}^{s}(v, v) \geq C_{1}\left\|e_{33}(v)\right\|_{L^{2}(\Omega)}^{2}=C_{1}\|v\|_{V}^{2} \text { (ellipticity). }
\end{aligned}
$$

The existence and uniqueness of solution to the family of bone remodeling rod models defined by (1.9) or (1.18) can be proved using the same arguments of Figueiredo and Trabucho [5] and also Monnier and Trabucho [9]. The proof of existence relies on Schauder's fixed point theorem together with the Cauchy-Lipschitz-Picard theorem (used to solve the remodeling rate equation, for a fixed dispacement), the Stampacchia theorem (that is necessary to guarantee the existence of solution to the variational inequality, for a fixed change of volume fraction) and regularity results. The proof of uniqueness is based on arguments similar to those of Cowin and Nachlinger [3]. The next theorem summarizes this statement of existence and uniqueness. 
Theorem 1.1 (Solution of (1.9)). Let $s \in[0, \delta]$ and we assume that, for each fixed $\hat{d}^{s}$, the unique solution $\hat{u}^{s}$ of the equilibrium problem

$$
\left\{\begin{array}{l}
\text { Find } \hat{u}^{s}(., t) \in K \subset V, \quad \text { such that: } \\
a_{\hat{d}^{s}}\left(\hat{u}^{s}, v-\hat{u}^{s}\right) \geq\left\langle L_{\hat{d}^{s}}, v-\hat{u}^{s}\right\rangle, \quad \forall v \in K,
\end{array}\right.
$$

has components with the regularity $\hat{u}_{\alpha}^{s}(., t) \in W_{0}^{2,2}(] 0, L[) \cap W^{3,2}(] 0, L[)$ and $\underline{\hat{u}}_{3}^{s}(., t) \in W_{0}^{1,2}(] 0, L[) \cap W^{2,2}(] 0, L[)$, for any $t \in[0, T]$ (which implies that $\hat{u}^{s}(., t) \in W^{2,2}(\Omega)$ ). Then, there exists a unique pair $\left(u^{s}, d^{s}\right)$ solution of problem (1.9), verifying

$$
u^{s} \in C^{1}([0, T] ; V) \quad \text { and } \quad d^{s} \in C^{1}\left([0, T] ; C^{0}(\bar{\Omega})\right) .
$$

\section{Partial Proof of Conditions (0.2) and (0.3)}

We prove in this section that the conditions (0.2) and (0.3) are satisfied for sub-families $\left\{A_{s_{j}}\right\}_{j=1}^{\infty}$ and $\left\{L_{s_{j}}\right\}_{j=1}^{\infty}$, where $s_{j} \in[0, \delta]$, of $\left\{A_{s}\right\}_{s>0}$ and $\left\{L_{s}\right\}_{s>0}$. Then, in Section 5 we conclude that (0.2) and (0.3) are true for the entire families $\left\{A_{s}\right\}_{s>0}$ and $\left\{L_{s}\right\}_{s>0}$.

Theorem 2.1. Let $\left(u^{s}, d^{s}\right)$ and $\left(u^{0}, d^{0}\right)$ be the solutions of problem (1.9) and (1.18), respectively. We assume that the conditions (1.7) are verified, and, for each $s, \xi_{0}^{s}=\xi_{0}, f_{i}^{s}=f_{i}, g_{i}^{s}=g_{i}$, where $\xi_{0}$, $f_{i}$ and $g_{i}$ are independent of $s$. Moreover we suppose that there exists a constant $c>0$, such that $\left\|u^{s}\right\|_{C^{0}\left([0, T] ; W^{2,2}(\Omega)\right)} \leq c$, for all $s \in[0, \delta]$. Then, for each $t$, there exists a subsequence of $\left\{\left(u^{s}, d^{s}\right)(., t)\right\}$, denoted by $\left\{\left(u^{s_{j}}, d^{s_{j}}\right)(., t)\right\}$, and elements $\bar{u}(., t) \in V$ and $\bar{d}(., t) \in L^{2}(\Omega)$, such that, when $s_{j} \rightarrow 0^{+}$

$$
\begin{aligned}
& \frac{u^{s_{j}}-u^{0}}{s_{j}}(., t) \rightarrow \bar{u}(., t) \quad \text { weakly in } \quad V, \\
& e_{33}\left(\frac{u^{s_{j}}-u^{0}}{s_{j}}\right)(., t) \rightarrow e_{33}(\bar{u})(., t) \quad \text { weakly in } L^{2}(\Omega), \\
& \frac{d^{s_{j}}-d^{0}}{s_{j}}(., t) \rightarrow \bar{d}(., t) \quad \text { weakly in } \quad L^{2}(\Omega), \\
& \left(u^{s_{j}}-u^{0}\right)(., t) \longrightarrow 0 \quad \text { strongly in } \quad V, \\
& e_{33}\left(u^{s_{j}}-u^{0}\right)(., t) \longrightarrow 0 \quad \text { strongly in } \quad L^{2}(\Omega), \\
& \left(d^{s_{j}}-d^{0}\right)(., t) \longrightarrow 0 \quad \text { strongly in } \quad L^{2}(\Omega), \\
& e_{33}\left(u^{s_{j}}-u^{0}\right) \longrightarrow 0 \quad \text { strongly in } \quad C^{0}\left([0, T] ; C^{0}(\bar{\Omega})\right), \\
& d^{s_{j}}-d^{0} \longrightarrow 0 \quad \text { strongly in } \quad C^{0}\left([0, T] ; C^{0}(\bar{\Omega})\right) .
\end{aligned}
$$

In addition the limit $\bar{d}$ depends implicity on $\bar{u}$ and is the solution of the following ordinary differential equation with respect to time

$$
\left[\begin{array}{l}
\dot{\bar{d}}=c\left(d^{0}\right) e_{33}(\bar{u})+\bar{d}\left[c^{\prime}\left(d^{0}\right) e_{33}\left(u^{0}\right)+b^{\prime}\left(d^{0}\right)\right]-c\left(d^{0}\right) \theta_{\alpha} \partial_{33} u_{\alpha}^{0} \\
\bar{d}(x, 0)=0, \quad \text { in } \bar{\Omega} .
\end{array}\right.
$$

Proof. The proof consists of four steps. The first two steps are preliminary results that prepare the proof of (2.1)-(2.8) in steps 3 and 4. 
Step 1. There exist positive constants $c_{1}$ and $c_{2}$ independent of $s$, such that

$$
\begin{aligned}
& \left\|u^{s}\right\|_{C^{0}([0, T] ; V)} \leq\left\|u^{s}\right\|_{C^{0}\left([0, T] ; W^{2,2}(\Omega)\right)} \leq c_{1}, \quad \forall s \in[0, \delta] \\
& \left\|d^{s}\right\|_{C^{0}\left([0, T] ; L^{2}(\Omega)\right)} \leq c_{2}, \quad \forall s \in[0, \delta] .
\end{aligned}
$$

The estimate (2.10) is a consequence of the hypotheses. Then, taking the integral, with respect to time in the remodeling rate equation we get

$$
d^{s}(x, t)=\int_{0}^{t}\left[c\left(d^{s}\right) e_{33}\left(u^{s}\right)+b\left(d^{s}\right)-s c\left(d^{s}\right) \theta_{\alpha} \partial_{33} u_{\alpha}^{s}\right](x, r) \mathrm{d} r+\bar{d}(x)
$$

Then we immediately deduce (2.11) taking the $L^{2}(\Omega)$ norm in the last equation and using (2.10) and (1.7).

Step 2. There exist positive constants $c_{3}$ and $c_{4}$ independents of $s$, such that

$$
\begin{aligned}
& \left\|\frac{u^{s}-u^{0}}{s}\right\|_{C^{0}([0, T] ; V)} \leq c_{3}, \quad \forall s \in[0, \delta], \\
& \left\|\frac{d^{s}-d^{0}}{s}\right\|_{C^{0}\left([0, T] ; L^{2}(\Omega)\right)} \leq c_{4}, \quad \forall s \in[0, \delta] .
\end{aligned}
$$

Choosing $v=u^{0}$ in problem (1.9) and $v=u^{s}$ in problem (1.18) and subtracting the two corresponding variational inequalities we obtain

$$
a_{d^{s}}\left(u^{s}, u^{s}-u^{0}\right)-a_{d^{0}}\left(u^{0}, u^{s}-u^{0}\right) \leq L_{d^{s}}\left(u^{s}-u^{0}\right)-L_{d^{0}}\left(u^{s}-u^{0}\right) .
$$

Dividing by $s^{2}$ and using the definitions of $a_{d^{s}}(.,$.$) and L_{d^{s}}$ we have

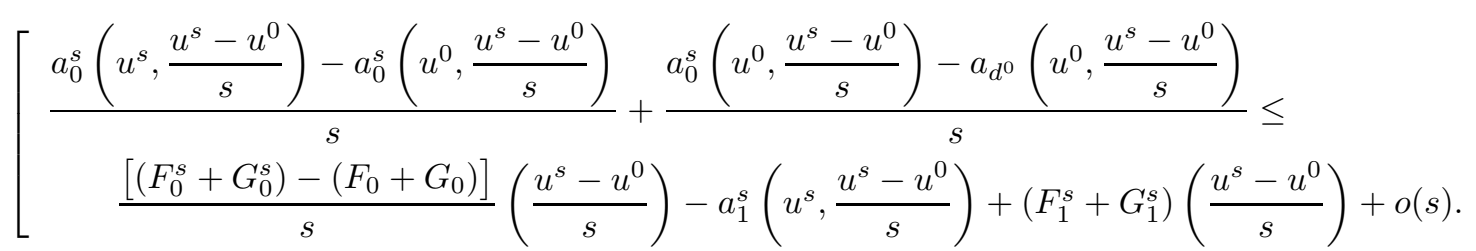

Now using the estimates $(2.10)-(2.11)$, the last inequality yields, for each $t \in[0, T]$

$$
\left\{\begin{array}{l}
a_{0}^{s}\left(\frac{u^{s}-u^{0}}{s}(., t), \frac{u^{s}-u^{0}}{s}(., t)\right) \leq \\
c\left\|\frac{u^{s}-u^{0}}{s}(., t)\right\|_{V}+\bar{c}\left\|\frac{d^{s}-d^{0}}{s}(., t)\right\|_{L^{2}(\Omega)}\left\|\frac{u^{s}-u^{0}}{s}(., t)\right\|_{V}+o(s),
\end{array}\right.
$$

where $c$ and $\bar{c}$ are positive constants independent of $s$ and $t$, and $|o(s)| \rightarrow 0$, as $s \rightarrow 0^{+}$. Consequently, because of the ellipticity of $a_{0}^{s}(.,),. c f$. (1.24), we have

$$
\left\|\frac{u^{s}-u^{0}}{s}(., t)\right\|_{V} \leq c\left\|\frac{d^{s}-d^{0}}{s}(., t)\right\|_{\left.L^{2}(\Omega)\right)}+\bar{c}
$$


where $c$ and $\bar{c}$ are other positive constants independent of $s$ and $t$. But subtracting the two remodeling rate equations in problems (1.9) and (1.18), and taking the integral with respect to time we obtain

$$
\left\{\begin{array}{c}
\left(d^{s}-d^{0}\right)(x, t)=\int_{0}^{t}\left[c\left(d^{s}\right) e_{33}\left(u^{s}-u^{0}\right)+\left[c\left(d^{s}\right)-c\left(d^{0}\right)\right] e_{33}\left(u^{0}\right)\right. \\
\left.+\left[b\left(d^{s}\right)-b\left(d^{0}\right)\right]-s c\left(d^{s}\right) \theta_{\alpha} \partial_{33} u_{\alpha}^{s}\right](x, r) \mathrm{d} r
\end{array}\right.
$$

and therefore, using (1.7), the mean value theorem for the terms $c\left(d^{s}\right)-c\left(d^{0}\right)$ and $b\left(d^{s}\right)-b\left(d^{0}\right)$, and dividing by $s$, we obtain

$$
\left\|\frac{d^{s}-d^{0}}{s}(., t)\right\|_{L^{2}(\Omega)} \leq \int_{0}^{t}\left[c_{1}\left\|\frac{u^{s}-u^{0}}{s}(., r)\right\|_{V}+c_{2}\left\|\frac{d^{s}-d^{0}}{s}(., r)\right\|_{L^{2}(\Omega)}+c_{3}\right] \mathrm{d} r
$$

where $c_{1}, c_{2}$ and $c_{3}$ are other positive constants independent of $s$ and $t$. Using now (2.18) and the integral Gronwall's inequality ( $c f$. Evans [4], p. 625) we have (2.14). Then, the property (2.13) is a consequence of (2.14) and (2.18).

Step 3. Because of the norm estimates (2.13)-(2.14) we directly obtain the weak convergences (2.1)-(2.3). The strong convergences (2.4)-(2.6) are a consequence of these weak convergences.

The strong convergence $(2.7)$ is a consequence of $(2.5)$ and the fact that $\partial_{3}\left(\underline{u}_{3}^{s_{j}}-\underline{u}_{3}^{0}\right)$ and $\partial_{33}\left(u_{\alpha}^{s_{j}}-u_{\alpha}^{0}\right)$ are bounded in the space $C^{0}\left([0, T] ; W^{1,2}(] 0, L[)\right)$ and $W^{1,2}(] 0, L[)$ is compactly imbedded in $C^{0}([0, L])$.

Taking into account the definition of $d^{s_{j}}-d^{0}$ given by $(2.19)$, the strong convergence (2.8) is a consequence of (2.7) and the integral's Gronwall inequality.

Step 4. To prove (2.9) we consider in (2.19) $s=s_{j}$ and we divide by $s_{j}$. Then for each $t$, when $s_{j} \rightarrow 0^{+}$

$$
\left\{\begin{array}{l}
c\left(d^{s_{j}}\right)(., t) \longrightarrow c\left(d^{0}\right)(., t) \quad \text { strongly in } C^{0}(\bar{\Omega}) \\
e_{33}\left(\frac{u^{s_{j}}-u^{0}}{s_{j}}\right)(., t) \rightarrow e_{33}(\bar{u})(., t) \quad \text { weakly in } L^{2}(\Omega), \\
\frac{c\left(d^{s_{j}}\right)-c\left(d^{0}\right)}{s_{j}} e_{33}\left(u^{0}\right)(., t) \rightarrow \bar{d} c^{\prime}\left(d^{0}\right) e_{33}\left(u^{0}\right)(., t) \quad \text { weakly in } L^{2}(\Omega), \\
\frac{b\left(d^{s_{j}}\right)-b\left(d^{0}\right)}{s_{j}}(., t) \rightarrow \bar{d} b^{\prime}\left(d^{0}\right)(., t) \quad \text { weakly in } \quad L^{2}(\Omega), \\
\partial_{33} u_{\alpha}^{s_{j}}(., t) \longrightarrow \partial_{33} u_{\alpha}^{0}(., t) \quad \text { strongly in } L^{2}(\Omega) .
\end{array}\right.
$$

Hence we conclude that, for each $t$, and for all $v \in L^{2}(\Omega)$

$$
\left\{\begin{aligned}
& \lim _{s_{j} \rightarrow 0^{+}} \int_{\Omega} \frac{d^{s_{j}}-d^{0}}{s}(., t) v \mathrm{~d} \Omega=\int_{\Omega}\left(\int _ { 0 } ^ { t } \left[c\left(d^{0}\right) e_{33}(\bar{u})\right.\right. \\
&\left.\left.+\bar{d} c^{\prime}\left(d^{0}\right) e_{33}\left(u^{0}\right)+\bar{d} b^{\prime}\left(d^{0}\right)-c\left(d^{0}\right) \theta_{\alpha} \partial_{33} u_{\alpha}^{0}\right](x, r) \mathrm{d} r\right) v \mathrm{~d} \Omega .
\end{aligned}\right.
$$

Therefore $\bar{d}(., t)$ must verify $(2.9)$, since the weak limit is unique. 
Theorem 2.2. With the hypotheses of the previous Theorem 2.1, there exist $A^{\prime}=A_{\bar{d}}^{\prime}$ and $L^{\prime}=L_{\bar{d}}^{\prime}$ depending explicitly on $\bar{d}$ and verifying, respectively, the conditions (0.2) and (0.3) for $s=s_{j}$, that is,

$$
\begin{aligned}
& \lim _{s_{j} \rightarrow 0^{+}}\left\|\left(\frac{A_{s_{j}}-A_{0}}{s_{j}}-A^{\prime}\right)(., t)\right\|_{\mathcal{L}\left(V ; V^{\prime}\right)}=0, \\
& \lim _{s_{j} \rightarrow 0^{+}}\left\|\left(\frac{L_{s_{j}}-L_{0}}{s_{j}}-L^{\prime}\right)(., t)\right\|_{V^{\prime}}=0 .
\end{aligned}
$$

For any $u$ and $v$ in $V, A^{\prime}(., t) \in \mathcal{L}\left(V^{\prime}, V\right)$ is defined by

$$
\left[\begin{array}{rl}
\left\langle A^{\prime} u, v\right\rangle= & -\int_{\Omega} b_{3333}^{\prime}\left(d^{0}\right) \frac{1}{b_{3333}^{2}\left(d^{0}\right)} \bar{d} e_{33}(u) e_{33}(v) \mathrm{d} \Omega \\
& +\int_{\Omega} \frac{-\theta_{\alpha}}{b_{3333}\left(d^{0}\right)}\left(e_{33}(u) \partial_{33} v_{\alpha}+e_{33}(v) \partial_{33} u_{\alpha}\right) \mathrm{d} \Omega \\
& +\int_{\Omega} \frac{1}{b_{3333}\left(d^{0}\right)} e_{33}(u) e_{33}(v) \operatorname{div} \theta \mathrm{d} \Omega
\end{array}\right.
$$

where $b_{3333}^{\prime}$ is the first derivative of the scalar function $b_{3333}$. The element $L^{\prime}(., t) \in V^{\prime}$ is defined by

$$
\left[\begin{array}{rl}
L^{\prime}(v)= & \int_{\Omega} \gamma \bar{d} P_{\eta}^{\prime}\left(d^{0}\right)\left(f_{\alpha} v_{\alpha}+f_{3} \underline{v}_{3}\right) \mathrm{d} \Omega \\
& +\int_{\Omega} \gamma\left(\xi_{0}+P_{\eta}\left(d^{0}\right)\right)\left[\left(f_{\alpha} v_{\alpha}+f_{3} \underline{v}_{3}\right) d i v \theta-f_{3} \theta_{\alpha} \partial_{3} v_{\alpha}\right] \mathrm{d} \Omega \\
& +\int_{\Gamma_{g}}\left[\left(g_{\alpha} v_{\alpha}+g_{3} \underline{v}_{3}\right) G_{1}(\theta, n)-g_{3} \theta_{\alpha} \partial_{3} v_{\alpha}\right] \mathrm{d} \Gamma
\end{array}\right.
$$

for any $v$ in $V$, where $P_{\eta}^{\prime}$ is the first derivative of the scalar function $P_{\eta}$.

Proof. We consider in the sequel $s=s_{j}$. Using the definitions of $A_{s}$ and $A_{0}$ we obtain

$$
\left[\begin{array}{l}
\frac{\left\langle A_{s} u, v\right\rangle-\left\langle A_{0} u, v\right\rangle}{s}= \\
\frac{a_{d^{s}}(u, v)-a_{d^{0}}(u, v)}{s}=\frac{a_{0}^{s}(u, v)-a_{d^{0}}(u, v)}{s}+a_{1}^{s}(u, v)+o(s),
\end{array}\right.
$$

where $|o(s)|$ tends to zero when $s \rightarrow 0^{+}$.

The calculus of the limit $a_{1}^{s}(u, v)$, when $s \rightarrow 0^{+}$is immediate. To compute the limit $\frac{a_{0}^{s}(u, v)-a_{d^{0}}(u, v)}{s}$, when $s \rightarrow 0^{+}$, we remark that the space $C_{0}^{\infty}([0, L])$ is dense in $H_{0}^{2}(] 0, L[)$ and $H_{0}^{1}(] 0, L[)$, for the norms $\|\cdot\|_{H^{2}}(] 0, L[)$ and $\|\cdot\|_{H^{1}(] 0, L[)}$, respectively. So by density, we only prove (2.25) for $u \in V$ and $v=\left(v_{1}, v_{2}, \underline{v}_{3}-x_{\alpha} \partial_{3} v_{\alpha}\right) \in V$, 
such that, $v_{\alpha} \in C_{0}^{\infty}([0, L])$ and $\underline{v}_{3} \in C_{0}^{\infty}([0, L])$. Thus, for each $t \in[0, T]$, when $s \rightarrow 0^{+}$, we obtain

$$
\left[\begin{array}{c}
\frac{a_{0}^{s}(u, v)-a_{d^{0}}(u, v)}{s}= \\
\int_{\Omega}\left(\frac{1}{b_{3333}\left(d^{s}\right)}-\frac{1}{b_{3333}\left(d^{0}\right)}\right) e_{33}(u) e_{33}(v) \mathrm{d} \Omega= \\
\int_{\Omega} \frac{b_{3333}\left(d^{0}\right)-b_{3333}\left(d^{s}\right)}{d^{s}-d^{0}}\left(b_{3333}\left(d^{s}\right) b_{3333}\left(d^{0}\right)\right)^{-1} \frac{d^{s}-d^{0}}{s} e_{33}(u) e_{33}(v) \mathrm{d} \Omega \\
\downarrow \\
-\int_{\Omega} b_{3333}^{\prime}\left(d^{0}\right) b_{3333}\left(d^{0}\right)^{-2} \bar{d} e_{33}(u) e_{33}(v) \mathrm{d} \Omega,
\end{array}\right.
$$

because $e_{33}(u) e_{33}(v) \in L^{2}(\Omega)$,

$$
\frac{b_{3333}\left(d^{0}\right)-b_{3333}\left(d^{s}\right)}{d^{s}-d^{0}}(., t) \longrightarrow b_{3333}^{\prime}\left(d^{0}\right)(., t), \quad \text { in } \quad C^{0}(\bar{\Omega}),
$$

and $\frac{d^{s}-d^{0}}{s}(., t)$ converges weakly to $\bar{d}(., t)$ in $L^{2}(\Omega)$. Therefore $(2.25)$ is proved.

Applying the definitions of $L_{d^{s}}$ and $L_{d^{0}}$ we get

$$
\left[\begin{array}{l}
\frac{L_{d^{s}}(v)-L_{d^{0}}(v)}{s}= \\
\frac{F_{0}^{s}(v)+G_{0}^{s}(v)-F_{0}(v)-G_{0}(v)}{s}+F_{1}^{s}(v)+G_{1}^{s}(v)+o(s),
\end{array}\right.
$$

where $|o(s)|$ tends to zero when $s \rightarrow 0^{+}$. So we obtain (2.26) by taking the limit in the latter expressions when $s \rightarrow 0^{+}$, using the definitions of $F_{0}^{s}, G_{0}^{s}, F_{1}^{s}, G_{1}^{s}$, and remarking that

$$
\frac{F_{0}^{s}+G_{0}^{s}-F_{0}-G_{0}}{s}(v) \longrightarrow \int_{\Omega} \gamma \bar{d} P_{\eta}^{\prime}\left(d^{0}\right)\left(f_{\alpha} v_{\alpha}+f_{3} \underline{v}_{3}\right) \mathrm{d} \Omega .
$$

So we conclude that the conditions (0.2) and (0.3) are proved for $s=s_{j}$.

\section{Proof of Condition (0.5)}

We show that condition (0.5) is verified, using a technique described in Sokolowski and Zolesio [12] p. 209, that consists in proving the polyhedricity of a modified constraint displacement set, and assuming an appropriate additional condition imposed to a non-negative Radon measure, as indicated in Proposition 3.4.

We consider the closed and convex subset $S$ of $H_{0}^{2}(] 0, L[)$ defined by

$$
S=\left\{\varphi \in H_{0}^{2}(] 0, L[): \quad \varphi\left(x_{3}\right) \geq \psi\left(x_{3}\right) \quad \text { in } \quad[0, L]\right\}
$$

and the operator

$$
\begin{array}{llll}
R: & V & \longrightarrow & H_{0}^{2}(] 0, L[) \\
& v=\left(v_{1}, v_{2}, v_{3}\right) & \longrightarrow & R(v)=v_{1} .
\end{array}
$$

It is clear that the constraint set $K$ verifies

$$
K=\{v \in V: \quad R(v) \in S\} .
$$


Moreover since $R$ maps $V$ onto $H_{0}^{2}(] 0, L[)$ and $0 \in S \subset H_{0}^{2}(] 0, L[)$, we have $\operatorname{Ker} R=\operatorname{Ker} R \cap K$, where $\operatorname{Ker} R=$ $\{v \in V: R v=0\}$. In addition $V=\operatorname{Ker} R \oplus(\operatorname{Ker} R)^{\perp}$, where $(\operatorname{Ker} R)^{\perp}=\left\{v \in V: a_{d^{0}}(v, u)=0, \forall u \in \operatorname{Ker} R\right\}$. The next proposition defines the operator $R^{-1} \in \mathcal{L}\left(H_{0}^{2}(] 0, L[),(\operatorname{Ker} R)^{\perp}\right)$, which is the right inverse of $R$, that is, $R \circ R^{-1}=i d_{H_{0}^{2}(] 0, L[)}$.

Proposition 3.1. The operator $R^{-1}$ is defined by

$$
R^{-1}(\varphi)=\left(\varphi, v_{2}, \underline{v}_{3}-x_{1} \partial_{3} \varphi-x_{2} \partial_{3} v_{2}\right)=v+u, \quad \forall \varphi \in H_{0}^{2}(] 0, L[),
$$

where $v=\left(0, v_{2}, \underline{v}_{3}-x_{2} \partial_{3} v_{2}\right)$ is the element of KerR solution of the equation

$$
a_{d^{0}}(v, z)=-a_{d^{0}}(u, z), \quad \forall z \in \operatorname{Ker} R,
$$

and $u=\left(\varphi, 0,-x_{1} \partial_{3} \varphi\right)$.

Proof. We define $R^{-1}(\varphi)$ by (3.4), because $R \circ R^{-1}(\varphi)=\varphi$ and $R^{-1}(\varphi)$ must be in $V$. Moreover, as $R^{-1}(\varphi)$ must be in $(\operatorname{Ker} R)^{\perp}$ we impose

$$
a_{d^{0}}\left(R^{-1}(\varphi), z\right)=0, \quad \forall z \in \operatorname{Ker} R .
$$

This is equivalent to find a $v=\left(0, v_{2}, \underline{v}_{3}-x_{2} \partial_{3} v_{2}\right) \in \operatorname{Ker} R$, such that $a_{d^{0}}(v+u, z)=0$, for all $z \in \operatorname{Ker} R$, where $u=\left(\varphi, 0,-x_{1} \partial_{3} \varphi\right)$. Hence (3.5) is an immediate consequence of the linearity of $a_{d^{0}}(.,$.$) with respect to$ the first component.

Obviously we can define a scalar product $((.,)$.$) in H_{0}^{2}(] 0, L[)$ in the following way

$$
((\zeta, \xi))=a_{d^{0}}\left(R^{-1} \zeta, R^{-1} \xi\right), \quad \forall \zeta, \xi \in H_{0}^{2}(] 0, L[),
$$

and the orthogonal projection $P_{S}$ associated to this new scalar product is defined by

$$
\begin{aligned}
P_{S}: H_{0}^{2}(] 0, L[) & \rightarrow S \subset H_{0}^{2}(] 0, L[) \\
\xi & \rightarrow P_{S}(\xi)
\end{aligned}
$$

where $\varphi=P_{S}(\xi)$ is the unique solution of the following variational inequality

$$
\left[\begin{array}{l}
\varphi=P_{S}(\xi) \in S: \\
((\varphi-\xi, \zeta-\varphi)) \geq 0, \quad \forall \zeta \in S .
\end{array}\right.
$$

Then, accordingly to Sokolowski and Zolesio [12], p. 209, for each $t \in[0, T]$, the unique solution $\Pi\left(L_{d^{0}}\right)(., t)=$ $u^{0}(., t)$ of the variational inequality

$$
\left[\begin{array}{l}
u^{0}(., t) \in K \subset V, \\
a_{d^{0}}\left(u^{0}, v-u^{0}\right) \geq\left\langle L_{d^{0}}, v-u^{0}\right\rangle, \quad \forall v \in K,
\end{array}\right.
$$

of problem (1.18), satisfies

$$
\left[\begin{array}{l}
\Pi\left(L_{d^{0}}\right)(., t)=\Upsilon\left(L_{d^{0}}\right)(., t)+R^{-1} P_{S}\left(\Phi\left(L_{d^{0}}\right)\right)(., t), \\
\text { with } \Upsilon\left(L_{d^{0}}\right)(., t) \in \operatorname{Ker} R, \quad \text { and } \quad R^{-1} P_{S}\left(\Phi\left(L_{d^{0}}\right)\right)(., t) \in(\operatorname{Ker} R)^{\perp} .
\end{array}\right.
$$

For any $l \in V^{\prime}$, the operator $\Upsilon: V^{\prime} \rightarrow \operatorname{Ker} R$ is defined by

$$
\left[\begin{array}{l}
\Upsilon(l) \in K \operatorname{Ker} R \subset V: \\
a_{d^{0}}(\Upsilon(l), z)=\langle l, z\rangle, \quad \forall z \in \operatorname{Ker} R \subset V,
\end{array}\right.
$$


and the operator $\Phi: V^{\prime} \rightarrow H_{0}^{2}(] 0, L[)$ is defined as follows

$$
\left[\begin{array}{l}
\Phi(l) \in H_{0}^{2}(] 0, L[): \\
((\Phi(l), \varphi))=\left\langle l, R^{-1} \varphi\right\rangle, \quad \forall \varphi \in H_{0}^{2}(] 0, L[) .
\end{array}\right.
$$

Due to the decomposition (3.11) and also because the mappings $\Upsilon, R^{-1}$ and $\Phi$ are linear and continuous we immediately conclude that, for each $t \in[0, T], \Pi$ is conically differentiable at $L_{d^{0}}(., t), c f$. (0.5), if and only if, $P_{S}$ is conically differentiable at $\Phi\left(L_{d^{0}}\right)(., t)$.

We prove now that the orthogonal projection $P_{S}$, with respect to the scalar product $((.,)$.$) defined in (3.7)$, is conical differentiable.

It is well known that the polyhedricity of the set $S$ at a given point $\varphi \in S$ implies the conical differentiability of $P_{S}$ at $\varphi$. For convenience of the reader we include in the paper the next statement, that recalls the definition of polyhedric set and the relation between polyhedricity and conical differentiability, applied to the set $S$ and the projection $P_{S}$ ( $c f$. Haraux [6], or Mignot [8], or Rao and Sokolowski [11]).

Proposition 3.2. The set $S \subset H_{0}^{2}(] 0, L[)$ is polyhedric at $\varphi \in S$, if for any $\xi \in H_{0}^{2}(] 0, L[)$, such that $\varphi=P_{S}(\xi)$ it follows

$$
T_{S}(\varphi) \cap[\varphi-\xi]^{\perp}=\overline{C_{S}(\varphi) \cap[\varphi-\xi]^{\perp}}
$$

where ${ }^{\perp}$ denotes the orthogonal with respect to the inner product $((.,)$.$) , the closure is in the space H_{0}^{2}(] 0, L[)$, $C_{S}(\varphi)$ is the convex cone defined by

$$
C_{S}(\varphi)=\left\{\zeta \in H_{0}^{2}(] 0, L[): \quad \exists_{r>0}, \quad \varphi\left(x_{3}\right)+r \zeta\left(x_{3}\right) \geq \psi\left(x_{3}\right) \quad \text { in } \quad\right] 0, L[\}
$$

and $T_{S}(\varphi)=\overline{C_{S}(\varphi)}$ is the tangent cone to $S$ at $\varphi \in S$, that is, the closure in the space $H_{0}^{2}([0, L[)$ of the convex cone $C_{S}(\varphi)$.

If condition (3.14) is satisfied, for a pair $(\varphi, \xi)$ in the space $H_{0}^{2}(] 0, L[) \times H_{0}^{2}(] 0, L[)$, with $\varphi=P_{S}(\xi)$, then for all $\zeta \in H_{0}^{2}(] 0, L[)$ and for $s>0$ small enough

$$
P_{S}(\xi+s \zeta)=P_{S}(\xi)+s P_{M}(\zeta)+o(s) \quad \text { and } \quad M=T_{S}(\varphi) \cap[\varphi-\xi]^{\perp}
$$

where $P_{M}$ is the orthogonal projection on $M$, and $\|o(s)\|_{H^{2}(] 0, L[)} / s \rightarrow 0$ as $s \rightarrow 0$. The condition (3.16) means that $P_{S}$ is conical differentiable at $\varphi \in S$.

Thus to conclude that $P_{S}$ is conical differentiable at a point $\varphi \in S$ it is enough to provide sufficient conditions under which the set $S$ is polyhedric at a point $\varphi \in S$. These sufficient conditions are summarized in the next proposition.

Proposition 3.3. The set $S$ is polyhedric at a point $\varphi \in S$, if the Radon measure $\mu$ defined by

$$
\left(\left(\varphi-P_{S}(\varphi), \zeta\right)\right)=-\int_{0}^{L} \zeta \mathrm{d} \mu, \quad \forall \zeta \in C_{0}^{\infty}(] 0, L[)
$$

is non-negative and its support denoted by supp $\mu$, that is a compact subset of $[0, L]$ and verifies supp $\mu \subset \Xi_{\psi}=$ $\left\{x_{3} \in\right] 0, L\left[: \varphi\left(x_{3}\right)=\psi\left(x_{3}\right)\right\}$, is admissible in the following sense

$$
\left\{\begin{array}{l}
\forall \zeta \in H_{0}^{2}(] 0, L[), \quad \text { such that } \quad \zeta=0 \quad C_{2}-\text { q.e on supp } \mu \\
\text { implies that } \left.\quad \zeta \in H_{0}^{2}(] 0, L \backslash \backslash \text { supp } \mu\right) .
\end{array}\right.
$$

In consequence the set $M$ defined in (3.16) is the following convex cone

$$
M=\left\{\zeta \in H_{0}^{2}(] 0, L[\backslash \text { supp } \mu): \quad \zeta\left(x_{3}\right) \geq 0, \quad C_{2}-\text { q.e. on } \Xi_{\psi}\right\} .
$$


(Note - we recall that a statement holds $C_{2}-q . e$. if it holds except for a set of $C_{2}$-capacity zero, where the $C_{2}$-capacity of a compact set $N, C_{2}(N)$, is defined by $C_{2}(N)=\inf \left\{\int_{0}^{L}\left|\partial_{33} \zeta\left(x_{3}\right)\right|^{2} \mathrm{~d} x_{3}: \quad \zeta \geq 1\right.$ on $N, \quad 0 \leq$ $\left.\left.\zeta \in C_{0}^{\infty}(] 0, L[)\right\}.\right)$

Proof. We first prove the two following statements i) and ii):

i) the scalar product $((.,)$.$) is equivalent to the usual scalar product (.,$.$) defined in H_{0}^{2}(] 0, L[)$ by

$$
(\varphi, \xi)=\int_{0}^{L} \partial_{33} \varphi\left(x_{3}\right) \partial_{33} \xi\left(x_{3}\right) \mathrm{d} x_{3}, \quad \forall \varphi, \xi \in H_{0}^{2}(] 0, L[)
$$

ii) the Radon measure $\mu$ defined in (3.17) is non-negative.

To prove i) we show that the norms $\|\cdot\|_{a_{d^{0}}}$ and $\|\cdot\|_{H_{0}^{2}(] 0, L[)}$ associated to the scalar products $((.)$,$) and (.,.)$ defined by (3.7) and (3.20), respectively, are equivalent. For any $\varphi \in H_{0}^{2}(] 0, L[)$ we have (see Prop. 3.1)

$$
\|\varphi\|_{a_{d^{0}}}^{2}=a_{d^{0}}\left(R^{-1} \varphi, R^{-1} \varphi\right)=\int_{\Omega} \frac{1}{b_{3333\left(d^{0}\right)}}\left(\partial_{3} \underline{v}_{3}-x_{1} \partial_{33} \varphi-x_{2} \partial_{33} v_{2}\right)^{2} \mathrm{~d} \Omega
$$

where $v=\left(0, v_{2}, \underline{v}_{3}-x_{2} \partial_{3} v_{2}\right) \in \operatorname{Ker} R$ is such that

$$
a_{d^{0}}(v, z)=-a_{d^{0}}(u, z), \quad \forall z \in \operatorname{KerR}
$$

with $u=\left(\varphi, 0,-x_{1} \partial_{3} \varphi\right)$, and thus

$$
\|\varphi\|_{a_{d^{0}}}^{2}=a_{d^{0}}(u+v, u+v)=a_{d^{0}}(u, u)+2 a_{d^{0}}(u, v)+a_{d^{0}}(v, v) .
$$

Choosing $z=v$ in (3.22) and using condition (1.7) we obtain

$$
\begin{aligned}
\|\varphi\|_{a_{d^{0}}}^{2} & =-a_{d^{0}}(v, v)+a_{d^{0}}(u, u) \leq a_{d^{0}}(u, u) \\
& =\int_{\Omega} \frac{1}{b_{3333}\left(d^{0}\right)} x_{1}^{2}\left|\partial_{33} \varphi\right|^{2} \mathrm{~d} \Omega \leq c\|\varphi\|_{H_{0}^{2}(] 0, L[)}^{2}
\end{aligned}
$$

where $c$ is a positive constant. On the other hand, using again condition (1.7) and (1.24) we get

$$
\begin{aligned}
\|\varphi\|_{a_{d^{0}}}^{2} & \geq C_{1}\left\|e_{33}\left(R^{-1} \varphi\right)\right\|_{L^{2}(\Omega)}^{2} \\
& =C_{1}\left\|\partial_{3}\left(\underline{v}_{3}-x_{1} \partial_{3} \varphi-x_{2} \partial_{3} v_{2}\right)\right\|_{L^{2}(\Omega)}^{2} \\
& =C_{1}\left[\int_{\Omega}\left(\partial_{3} \underline{v}_{3}\right)^{2} \mathrm{~d} \Omega+\int_{\Omega} x_{1}^{2}\left(\partial_{33} \varphi\right)^{2} \mathrm{~d} \Omega+\int_{\Omega} x_{2}^{2}\left(\partial_{33} v_{2}\right)^{2} \mathrm{~d} \Omega\right] \\
& \geq C_{1} \int_{\Omega} x_{1}^{2}\left(\partial_{33} \varphi\right)^{2} \mathrm{~d} \Omega=C\left(\int_{\omega} x_{1}^{2} \mathrm{~d} \omega\right)\|\varphi\|_{H_{0}^{2}(] 0, L[)}^{2},
\end{aligned}
$$

where $C_{1}$ and $C$ represent different positive constants. Thus the proof of i) is complete.

To prove ii) it suffices to remark that for all $\zeta \in C_{0}^{\infty}(] 0, L[)$ such that $\zeta \geq 0$ in $] 0, L[$ we have

$$
\left\{\begin{array}{c}
\left(\left(\varphi-P_{S}(\varphi), \zeta\right)\right)=\left(\left(\varphi-P_{S}(\varphi), \zeta+P_{S}(\varphi)-P_{S}(\varphi)\right)\right) \\
=\left(\left(\varphi-P_{S}(\varphi), \xi-P_{S}(\varphi)\right)\right) \leq 0
\end{array}\right.
$$

because of the definition of $P_{S}(\varphi)$ and the fact that $\xi=\zeta+P_{S}(\varphi)$ belongs to $S$.

Due to the Properties i) and ii) and assuming that the set supp $\mu$ is admissible in the sense of (3.18), we finish the proof of this theorem, using exactly the same arguments as in Rao and Sokolowski [11]. 
The verification that the set supp $\mu$ is admissible in the sense of (3.18) is in general difficult (cf. also Pierre and Sokolowski [10] for the related subject of differentiability of projections and applications). Nevertheless there is a sufficient condition for which the supp $\mu$ is admissible, as described in the next Proposition 3.4 ( $c f$. Rao and Sokolowski [11] for the proof of this statement). Therefore assembling this last comment with Proposition 3.3, we can state the following result concerning the conical differentiability of the projection $P_{S}$ at a point $\varphi \in S$.

Proposition 3.4. If the support of the Radon measure $\mu$ defined in (3.17) is admissible in the sense of (3.18), then, the set $S$ is polyhedric at the point $\varphi \in S$, and, consequently, $P_{S}$ is conical differentiable at $\varphi \in S$. In particular, if the $C_{1}$-capacity of the compact set supp $\mu$ is zero, that is $C_{1}($ supp $\mu)=0$, where

$$
C_{1}(\operatorname{supp} \mu)=\inf \left\{\int_{0}^{L}\left|\partial_{3} \zeta\left(x_{3}\right)\right|^{2} \mathrm{~d} x_{3}: \quad \zeta \geq 1 \text { on } \operatorname{supp} \mu, \quad 0 \leq \zeta \in C_{0}^{\infty}(] 0, L[)\right\},
$$

then supp $\mu$ is admissible.

Finally, assuming the hypothesis of the previous proposition 3.4, and using the decomposition (3.11) and (3.16) we conclude that, for each $t \in[0, T]$, the operator $Q(., t)$ in $(0.5)$, which is the conical derivative of $\Pi$ at $L_{d^{0}}(., t)$, is defined by

$$
Q(l)(., t)=\Upsilon(l)(., t)+R^{-1} P_{M(., t)}(\Phi(l))(., t), \quad \forall l \in V^{\prime},
$$

where for each $t$, the convex cone $M(., t)$ depends on $L_{d^{0}}(., t)$ and the obstacle $\psi$, and is defined in (3.16) with $\varphi=\Phi\left(L_{d^{0}}\right)(., t)$, that is,

$$
M(., t)=T_{S}\left(\Phi\left(L_{d^{0}}\right)(., t)\right) \cap\left[\Phi\left(L_{d^{0}}\right)(., t)-\xi\right]^{\perp},
$$

where $\Phi\left(L_{d^{0}}\right)(., t)=P_{S}(\xi)$, for some $\xi \in H_{0}^{2}(] 0, L[)$.

\section{Partial Proof of Conditions (0.6) and (0.7)}

In this section we prove that conditions $(0.6)$ and (0.7) are satisfied for a sub-family $\left\{\left(u^{s_{j}}, d^{s_{j}}\right)\right\}_{j=1}^{\infty}$ of $\left\{\left(u^{s}, d^{s}\right)\right\}_{s>0}$. In Section 5 we show that these two conditions are still verified for the all family $\left\{\left(u^{s}, d^{s}\right)\right\}_{s>0}$.

By Theorem 2.1 we know that there exists a subsequence that we denote by $\left(\frac{u^{s_{j}}-u^{0}}{s_{j}}, \frac{d^{s_{j}}-d^{0}}{s_{j}}\right)(., t)$ that converges weakly to $(\bar{u}, \bar{d})(., t)$ in $V \times L^{2}(\Omega)$, when $s_{j} \rightarrow 0^{+}$. Consequently, by Theorems 2.1 and 2.2 , there exist $A^{\prime}=A_{\bar{d}}^{\prime}$ and $L^{\prime}=L_{\bar{d}}^{\prime}$ that depend explicitly on $\bar{d}$ and implicitly on $\bar{u}$. Using the Theorem 4.14 of Sokolowski and Zolesio [12] p. 178, combined with the expression (3.28) for $Q(., t)$ (or equivalently Th. 4.30 of Sokolowski and Zolesio [12] p. 210) we conclude that, for all $s_{j}$

$$
\left[\begin{array}{l}
u^{s_{j}}(., t)=u^{0}(., t)+s_{j} u^{\prime}(., t)+o\left(s_{j}\right), \quad \text { with } \\
u^{\prime}=Q\left(L_{\bar{d}}^{\prime}-A_{\bar{d}}^{\prime} u^{0}\right)=\Upsilon\left(L_{\bar{d}}^{\prime}-A_{\bar{d}}^{\prime} u^{0}\right)+R^{-1} P_{M}\left(\Phi\left(L_{\bar{d}}^{\prime}-A_{\bar{d}}^{\prime} u^{0}\right)\right)
\end{array}\right.
$$

where $\left\|o\left(s_{j}\right) / s_{j}\right\|_{V}$ tends to zero when $s_{j} \rightarrow 0^{+}$, and $\bar{d}$ is the solution of the following ordinary differential equation ( $c f$. Th. 2.1)

$$
\left[\begin{array}{l}
\dot{\bar{d}}=c\left(d^{0}\right) e_{33}(\bar{u})+\bar{d}\left[c^{\prime}\left(d^{0}\right) e_{33}\left(u^{0}\right)+b^{\prime}\left(d^{0}\right)\right]-c\left(d^{0}\right) \theta_{\alpha} \partial_{33} u_{\alpha}^{0} \\
\bar{d}(x, 0)=0, \quad \text { in } \bar{\Omega} .
\end{array}\right.
$$

Moreover from (4.1) and (2.1) we also conclude that $\bar{u}=u^{\prime}$. From (4.2) and (2.3) we deduce that $\bar{d}=d^{\prime}$ and

$$
d^{s_{j}}(., t)=d^{0}(., t)+s_{j} d^{\prime}(., t)+o\left(s_{j}\right),
$$

where $\frac{\int_{\Omega} o\left(s_{j}\right) v \mathrm{~d} \Omega}{s_{j}}$ tends to zero when $s_{j} \rightarrow 0^{+}$, for all $v \in L^{2}(\Omega)$. So the conditions $(0.6)$ and $(0.7)$ are proved for the subfamily of parameters $s=s_{j}$. 


\section{Proof of Theorem 0.1}

In this section we prove Theorem 0.1 with the hypotheses of Theorem 2.1 and the sufficient conditions of Proposition 3.3.

Observing (4.1), (4.2) and (4.3), and taking into account the results of Section 3 and also the Theorem 4.14 of Sokolowski and Zolesio [12] p. 178 (or equivalently Th. 4.30 of Sokolowski and Zolesio [12] p. 210), we realize that to prove conditions $(0.2)-(0.3)$ and $(0.6)-(0.7)$, and consequently to prove Theorem 0.1 , it only remains to assure that the weak limit $(\bar{u}, \bar{d})(., t)$ is unique. That is, for all $s>0$, the sequence $\left(\frac{u^{s}-u^{0}}{s}, \frac{d^{s}-d^{0}}{s}\right)(., t)$ converges weakly to $(\bar{u}, \bar{d})(., t) \in V \times L^{2}(\Omega)$. This happens if the system defined by the second equation in (4.1) and (4.2) has a unique solution. In fact this is true, as stated and proved in the next theorem.

Theorem 5.1. The system

$$
\left[\begin{array}{l}
\text { Find } \quad(u, d)(., t) \in V \times L^{2}(\Omega): \\
u=\Upsilon\left(L_{d}^{\prime}-A_{d}^{\prime} u^{0}\right)+R^{-1} P_{M}\left(\Phi\left(L_{d}^{\prime}-A_{d}^{\prime} u^{0}\right)\right) \\
\dot{d}=c\left(d^{0}\right) e_{33}(u)+d\left[c^{\prime}\left(d^{0}\right) e_{33}\left(u^{0}\right)+b^{\prime}\left(d^{0}\right)\right]-c\left(d^{0}\right) \theta_{\alpha} \partial_{33} u_{\alpha}^{0} \\
d(x, 0)=0, \quad \text { in } \quad \bar{\Omega}
\end{array}\right.
$$

has a unique solution $(u, d) \in C^{1}([0, T] ; V) \times C^{1}\left([0, T] ; C^{0}(\bar{\Omega})\right)$.

Proof. The proof of existence is analogous to the proof of Theorem 1.1. It relies on Schauder's fixed point theorem together with the Cauchy-Lipschitz-Picard theorem (used to solve the ordinary differential equation for a fixed $u$ ) and regularity results, concerning the first equation of (5.1). To prove that the solution of (5.1) is unique let $(u, d)$ and $(v, e)$ be two different solutions of $(5.1)$. Then we have

$$
\left[\begin{array}{rl}
u-v= & \Upsilon\left(L_{d}^{\prime}-L_{e}^{\prime}-\left(A_{d}^{\prime}-A_{e}^{\prime}\right) u^{0}\right) \\
& +R^{-1}\left[P_{M}\left(\Phi\left(L_{d}^{\prime}-A_{d}^{\prime} u^{0}\right)\right)+P_{M}\left(\Phi\left(L_{e}^{\prime}-A_{e}^{\prime} u^{0}\right)\right)\right]
\end{array}\right.
$$

Taking the norm in $V$ and using the continuity of the operators $\Upsilon, R^{-1}, P_{M}, \Phi$ and the linearity of $\Upsilon, R^{-1}$, $\Phi$, we obtain for each $t \in[0, T]$

$$
\|(u-v)(., t)\|_{V} \leq C\|(d-e)(., t)\|_{L^{2}(\Omega)},
$$

where $C$ is a positive constant. On the other hand, subtracting the two ordinary differential equations, and integrating in time

$$
\left[\begin{array}{l}
(d-e)(x, t)= \\
\int_{0}^{t}\left(c\left(d^{0}\right) e_{33}(u-v)+(d-e)\left[c^{\prime}\left(d^{0}\right) e_{33}\left(u^{0}\right)+b^{\prime}\left(d^{0}\right)\right]\right)(x, r) \mathrm{d} r
\end{array}\right.
$$

Taking the $L^{2}(\Omega)$, for each $t \in[0, T]$, and using (5.3) we get

$$
\|(d-e)(., t)\|_{L^{2}(\Omega)} \leq C \int_{0}^{t}\|(d-e)(., t)\|_{L^{2}(\Omega)}(x, r) \mathrm{d} r
$$

where $C$ is positive constant independent of $t$. Applying now to (5.5) the integral Gronwall's inequality we have that $d=e$ and by (5.3) also $u=v$, so the proof is complete. 


\section{REFERENCES}

[1] P.G. Ciarlet, Mathematical Elasticity, Vol. 1: Three-Dimensional Elasticity. Stud. Math. Appl., North-Holland, Amsterdam 20 (1988).

[2] S.C. Cowin and D.H. Hegedus, Bone remodeling I: theory of adaptive elasticity. J. Elasticity 6 (1976) 313-326.

[3] S.C. Cowin and R.R. Nachlinger, Bone remodeling III: uniqueness and stability in adaptive elasticity theory. J. Elasticity 8 (1978) 285-295.

[4] L.C. Evans, Partial Differential Equations. American Mathematical Society, Providence, Rhode Island (1998).

[5] I.N. Figueiredo and L. Trabucho, Asymptotic model of a nonlinear adaptive elastic rod. Math. Mech. Solids 9 (2004) 331-354.

[6] A. Haraux, How to differentiate the projection on a convex set in Hilbert space. Some applications to variational inequalities. J. Math. Soc. Japan 29 (1977) 615-631.

[7] D.H. Hegedus and S.C. Cowin, Bone remodeling II: small strain adaptive elasticity. J. Elasticity 6 (1976) 337-352.

[8] F. Mignot, Contrôle dans les inéquations variationnelles elliptiques. J. Funct. Anal. 22 (1976) 130-185.

[9] J. Monnier and L. Trabucho, An existence and uniqueness result in bone remodeling theory. Comput. Methods Appl. Mech. Engrg. 151 (1998) 539-544.

[10] M. Pierre and J. Sokolowski, Differentiability of projection and applications, E. Casas Ed. Marcel Dekker, New York. Lect. Notes Pure Appl. Math. 174 (1996) 231-240.

[11] M. Rao and J. Sokolowski, Sensitivity analysis of unilateral problems in $H_{0}^{2}(\Omega)$ and applications. Numer. Funct. Anal. Optim. 14 (1993) 125-143.

[12] J. Sokolowski and J.-P. Zolesio, Introduction to Shape Optimization, Shape Sensitivity Analysis. Springer-Verlag, New York, Springer Ser. Comput. Math. 16 (1992).

[13] L. Trabucho and J.M. Viaño, Mathematical Modelling of Rods, P.G. Ciarlet and J.L Lions Eds. North-Holland, Amsterdam, Handb. Numer. Anal. 4 (1996) 487-974.

[14] T. Valent, Boundary Value Problems of Finite Elasticity. Springer Tracts Nat. Philos. 31 (1988). 